\title{
POINTWISE COMPLETENESS AND POINTWISE DEGENERACY OF POSITIVE FRACTIONAL DESCRIPTOR CONTINUOUS-TIME LINEAR SYSTEMS WITH REGULAR PENCILS
}

\author{
TADEUSZ KACZOREK ${ }^{a}$ \\ ${ }^{a}$ Faculty of Electrical Engineering \\ Białystok University of Technology, ul. Wiejska 45D, 15-351 Białystok, Poland \\ e-mail:kaczorek@isep.pw.edu.pl
}

\begin{abstract}
Pointwise completeness and pointwise degeneracy of positive fractional descriptor continuous-time linear systems with regular pencils are addressed. Conditions for pointwise completeness and pointwise degeneracy of the systems are established and illustrated by an example.
\end{abstract}

Keywords: pointwise completeness, pointwise degeneracy, descriptor system, fractional system, positive system.

\section{Introduction}

Descriptor (singular) linear systems have been considered in many papers and books (Bru et al., 2003; 2002; Choundhury, 1972; Campbell et al., 1976; Dai, 1989; Guang-Ren Duan, 2010; Kaczorek, 2013; 2011a; 2014b; 2004; 1992; 2014c; 2011c; 2011d; 2011e; Virnik, 2008). The eigenvalue and invariant assignment by state and output feedbacks was investigated by Kaczorek (2004; 2011d), along with minimum energy control of descriptor linear systems (Kaczorek, 2014b). In positive systems inputs, state variables and outputs take only nonnegative values (Farina and Rinaldi, 2000; Kaczorek, 2002). Examples of positive systems are industrial processes involving chemical reactors, heat exchangers and distillation columns, storage systems, compartmental systems, water and atmospheric pollution models. A variety of models having positive linear behavior can be found in engineering, management science, economics, social sciences, biology and medicine, etc. Positive fractional linear systems and selected problems in the theory of fractional systems were addressed by Kaczorek (2011d).

Descriptor standard positive linear systems by the use of the Drazin inverse were discussed by Bru et al. (2003; 2002), Campbell et al. (1976) and Kaczorek (2013; 2011a; 2014a; 2011c; 2011e), who also applied the shuffle algorithm to check the positivity of descriptor linear systems (Kaczorek, 2011a). The stability of positive descriptor systems was investigated by Virnik (2008). Reduction and decomposition of descriptor fractional discrete-time linear systems were considered by Kaczorek (2011c), who also introduced a new class of descriptor fractional linear discrete-time systems (Kaczorek, 2011e). Pointwise completeness and pointwise degeneracy for standard and fractional linear systems were investigated by Busłowicz (2008), Busłowicz et al. (2006), Choundhury (1972), Kaczorek and Busłowicz (2009), Kaczorek (2011b; 2009; 2010), Olbrot (1972), Popov (1972), Trzasko et al. (2007) and Weiss (1970). The Drazin inverse of matrices was applied to find solutions of the state equations of fractional descriptor continuous-time linear systems with regular pencils by Kaczorek (2014a).

In this paper, pointwise completeness and pointwise degeneracy of positive fractional descriptor continuous-time linear systems with regular pencils will be addressed.

The paper is organized as follows. In Section 2 some definitions, lemmas and theorems concerning the positive fractional descriptor continuous-time linear systems are recalled. The main result of the paper is presented in Section 3, where the conditions for the pointwise completeness and pointwise degeneracy of fractional descriptor continuous-time linear systems with regular pencils are established and illustrated with an example. Concluding remarks are given in Section 4. 
The following notation will be used: $\mathbb{R}$, the set of real numbers; $\mathbb{R}^{n \times m}$, the set of $n \times m$ real matrices and $\mathbb{R}^{n}=\mathbb{R}^{n \times 1} ; \mathbb{R}_{+}^{n \times m}$, the set of $n \times m$ matrices with nonnegative entries and $\mathbb{R}_{+}^{n}=\mathbb{R}_{+}^{n \times 1} ; M_{n}$, the set of $n \times n$ Metzler matrices (real matrices with nonnegative off-diagonal entries); $I_{n}$, the $n \times n$ identity matrix, $\operatorname{ker} A$ (im $A$ ), the kernel (image) of the matrix $A$.

\section{Preliminaries}

Consider the autonomous fractional descriptor continuous-time linear system

$$
E_{0} D_{t}^{\alpha} x(t)=A x(t), \quad 0<\alpha<1,
$$

where $\alpha$ is the fractional order, $x(t) \in \mathbb{R}^{n}$ is the state vector, $E, A \in \mathbb{R}^{n \times n}$, and

$$
{ }_{0} D_{t}^{\alpha} x(t)=\frac{1}{\Gamma(1-\alpha)} \int_{0}^{t} \frac{1}{(t-\tau)^{\alpha}} \frac{\mathrm{d} x(\tau)}{\mathrm{d} \tau} \mathrm{d} \tau
$$

is the Caputo definition of the $\alpha$-th $(\alpha \in \mathbb{R})$ order derivative of $x(t)$, and

$$
\Gamma(\alpha)=\int_{0}^{\infty} e^{-t} t^{\alpha-1} \mathrm{~d} t
$$

is the Euler gamma function.

It is assumed that det $E=0$, but the pencil $(E, A)$ of (1) is regular, i.e.,

$$
\operatorname{det}[E s-A] \neq 0 \quad \text { for some } s \in \mathbb{C} \text {. }
$$

Assuming that, for some chosen $c \in \mathbb{C}$, $\operatorname{det}[E c-A] \neq 0$ and premultiplying (1) by $[E c-A]^{-1}$, we obtain

$$
\bar{E}_{0} D_{t}^{\alpha} x(t)=\bar{A} x(t),
$$

where

$$
\bar{E}=[E c-A]^{-1} E, \quad \bar{A}=[E c-A]^{-1} A .
$$

Note that Eqns. (1) and (5a) have the same solution $x(t)$.

Definition 1. (Campbell et al., 1976; Kaczorek, 1992) The smallest nonnegative integer $q$ is called the index of the matrix $\bar{E} \in \mathbb{R}^{n \times n}$ if

$$
\operatorname{rank} \bar{E}^{q}=\operatorname{rank} \bar{E}^{q+1} .
$$

Definition 2. (Campbell et al., 1976; Kaczorek, 1992) A matrix $\bar{E}^{D}$ is called the Drazin inverse of $\bar{E} \in \mathbb{R}^{n \times n}$ if it satisfies the conditions

$$
\begin{aligned}
\bar{E} \bar{E}^{D} & =\bar{E}^{D} \bar{E}, \\
\bar{E}^{D} \bar{E} \bar{E}^{D} & =\bar{E}^{D}, \\
\bar{E}^{D} \bar{E}^{q+1} & =\bar{E}^{q},
\end{aligned}
$$

where $q$ is the index of $\bar{E}$ defined by $[6$.
The Drazin inverse $\bar{E}^{D}$ of a square matrix $\bar{E}$ always exists and is unique (Campbell et al., 1976; Kaczorek, 1992). If $\operatorname{det} \bar{E} \neq 0$, then $\bar{E}^{D}=\bar{E}^{-1}$. Some methods for computation of the Drazin inverse are given by Kaczorek (1992).

Lemma 1. (Campbell et al., 1976; Kaczorek, 1992; 2014a) The matrices $\bar{E}$ and $\bar{A}$ defined by (5b) satisfy the following equalities:

$$
\begin{aligned}
& \bar{A} \bar{E}=\bar{E} \bar{A}, \quad \bar{A}^{D} \bar{E}=\bar{E} \bar{A}^{D}, \\
& \bar{E}^{D} \bar{A}=\bar{A} \bar{E}^{D}, \quad \bar{A}^{D} \bar{E}^{D}=\bar{E}^{D} \bar{A}^{D}, \\
& \operatorname{ker} \bar{A} \cap \operatorname{ker} \bar{E}=\{0\}, \\
& \bar{E}=T\left[\begin{array}{cc}
J & 0 \\
0 & N
\end{array}\right] T^{-1}, \\
& \bar{E}^{D}=T\left[\begin{array}{rr}
J^{-1} & 0 \\
0 & 0
\end{array}\right] T^{-1}, \\
& \operatorname{det} T \neq 0, J \in \mathbb{R}^{n_{1} \times n_{1}}, \text { is nonsingular } \\
& \text { is nilpotent, } n_{1}+n_{2}=n, \\
& \left(I_{n}-\bar{E} \bar{E}^{D}\right) \bar{A} \bar{A}^{D}=I_{n}-\bar{E} \bar{E}^{D}, \\
& \left(I_{n}-\bar{E} \bar{E}^{D}\right)\left(\bar{E} \bar{A}^{D}\right)^{q}=0 .
\end{aligned}
$$$$
\operatorname{det} T \neq 0, J \in \mathbb{R}^{n_{1} \times n_{1}} \text {, is nonsingular, } N \in \mathbb{R}^{n_{2} \times n_{2}}
$$

Theorem 1. (Kaczorek, 2014a) The solution of Eqn. (1) is given by

$$
x(t)=\Phi_{0}(t) \bar{E} \bar{E}^{D} w
$$

where

$$
\Phi_{0}(t)=\sum_{k=0}^{\infty} \frac{\left(\bar{E}^{D} \bar{A}\right)^{k} t^{k \alpha}}{\Gamma(k \alpha+1)}
$$

and the vector $w \in \mathbb{R}^{n}$ is arbitrary.

From (9a) we have $x(0)=x_{0}=\bar{E} \bar{E}^{D} w$ and $x_{0} \in$ $\operatorname{im}\left(\bar{E} \bar{E}^{D}\right)$, where 'im' denotes the image of $\bar{E} \bar{E}^{D}$.

Lemma 2. (Kaczorek, 2014a) The matrix $\Phi_{0}(t)$ defined by $(9 b)$ is nonsingular for any matrix $A \in \mathbb{R}^{n \times n}$ and time $t \geq 0$.

Theorem 2. (Kaczorek, 2014a) Let

$$
P=\bar{E} \bar{E}^{D} \quad \text { and } \quad Q=\bar{E}^{D} \bar{A} .
$$

Then we have

$$
\begin{aligned}
P^{k} & =P \quad \text { for } \quad k=2,3, \ldots, \\
P Q & =Q P=Q, \\
P \Phi_{0}(t) & =\Phi_{0}(t) .
\end{aligned}
$$$$
\text { (iii) }
$$

Definition 3. (Farina and Rinaldi, 2000; Kaczorek, 2011d) The fractional descriptor system (1) is called (internally) positive if the state vector $x(t) \in \mathbb{R}_{+}^{n}, t \geq 0$, for all initial conditions $x_{0} \in \mathbb{R}_{+}^{n}$.

Theorem 3. (Farina and Rinaldi, 2000; Kaczorek, 2011d) The fractional descriptor system (1) is (internally) positive if and only if

$$
\bar{E}^{D} \bar{A} \in M_{n} .
$$




\section{Pointwise completeness and pointwise degeneracy}

Definition 4. The positive fractional descriptor system (1) is called pointwise complete for $t=t_{f}$ if for every final state $x_{f} \in \mathbb{R}_{+}^{n}$ there exists a vector of initial conditions $x_{0} \in \operatorname{im}\left(\bar{E} \bar{E}^{D}\right) \in \mathbb{R}_{+}^{n}$ such that $x\left(t_{f}\right)=x_{f} \in \mathbb{R}_{+}^{n}$.

A matrix $A \in \mathbb{R}^{n \times n}$ is called monomial if in each row and in each column it has only one positive entry and the remaining entries are zero.

Theorem 4. The positive fractional descriptor system (1) is pointwise complete for any $t=t_{f}$ and every final state $x_{f} \in \mathbb{R}^{n}$ belonging to the set

$$
x_{f}=\operatorname{im}\left[\Phi_{0}\left(t_{f}\right) x_{0}\right] \in \mathbb{R}_{+}^{n}
$$

if and only if $\Phi_{0}\left(t_{f}\right) \in \mathbb{R}_{+}^{n \times n}$ is a monomial matrix.

Proof. Substituting in (9a) $t=t_{f}$, we obtain

$$
x_{f}=x\left(t_{f}\right)=\Phi_{0}\left(t_{f}\right) x_{0}
$$

and

$$
x_{0}=\left[\Phi_{0}\left(t_{f}\right)\right]^{-1} x_{f},
$$

since the matrix $\Phi_{0}(t)$ is monomial and

$$
\left[\Phi_{0}\left(t_{f}\right)\right]^{-1} \in \mathbb{R}_{+}^{n \times n} .
$$

Definition 5. The positive fractional descriptor system (1) is called pointwise degenerated in the direction $v$ for $t=$ $t_{f}$ if there exists a nonzero vector $v \in \mathbb{R}^{n}$ such that for all initial conditions $x_{0} \in \operatorname{im}\left(\bar{E} \bar{E}^{D}\right) \in \mathbb{R}_{+}^{n}$ the solution of (11) satisfies the condition

$$
v^{T} x_{f}=0
$$

where $T$ denotes the transpose.

Theorem 5. The positive fractional descriptor system (1) is pointwise degenerated in the direction $v$ defined by

$$
v^{T} \bar{E}=0
$$

for any $t_{f} \geq 0$ and all initial conditions $x_{0} \in$ $\operatorname{im}\left(\bar{E} \bar{E}^{D}\right) \in \mathbb{R}_{+}^{n}$.

Proof. Postmultiplying (17) by $\bar{E}^{D} w$ and using $x_{0}=$ $\bar{E} \bar{E}^{D} w$ and (16), we obtain

$$
v^{T} \bar{E} \bar{E}^{D} w=v^{T} x_{0}=0 .
$$

Taking into account (9b), (14) and (17), we obtain

$$
\begin{aligned}
v^{T} x_{f} & =v^{T} \Phi_{0}\left(t_{f}\right) x_{0} \\
& =\sum_{k=0}^{\infty} \frac{v^{T}\left(\bar{E}^{D} \bar{A}\right)^{k} t_{f}^{k \alpha}}{\Gamma(k \alpha+1)} x_{0} \\
& =v^{T} x_{0}+\sum_{k=1}^{\infty} \frac{v^{T} \bar{E} \bar{E}^{D}\left(\bar{E}^{D} \bar{A}\right)^{k} t_{f}^{k \alpha}}{\Gamma(k \alpha+1)} x_{0}=0,
\end{aligned}
$$

since (7b) and 17 hold.
Example 1. Consider the positive fractional descriptor system (1) with the matrices

$$
E=\left[\begin{array}{ll}
1 & 0 \\
0 & 0
\end{array}\right], \quad A=\left[\begin{array}{cc}
-1 & 0 \\
0 & -2
\end{array}\right] .
$$

The pencil of (20) is regular since

$$
\operatorname{det}[E s-A]=\left|\begin{array}{cc}
s+1 & 0 \\
0 & 2
\end{array}\right|=2(s+1) \neq 0
$$

and the assumption (4) is met.

Choosing $c=1$, we obtain

$$
\begin{aligned}
& \bar{E}=[E c-A]^{-1} E \\
& =\left[\begin{array}{ll}
2 & 0 \\
0 & 2
\end{array}\right]^{-1}\left[\begin{array}{ll}
1 & 0 \\
0 & 0
\end{array}\right] \\
& =\left[\begin{array}{cc}
0.5 & 0 \\
0 & 0
\end{array}\right] \text {, } \\
& \bar{A}=[E c-A]^{-1} A \\
& =\left[\begin{array}{ll}
2 & 0 \\
0 & 2
\end{array}\right]^{-1}\left[\begin{array}{cc}
-1 & 0 \\
0 & -2
\end{array}\right] \\
& =\left[\begin{array}{cc}
-0.5 & 0 \\
0 & -1
\end{array}\right]
\end{aligned}
$$

and

$$
\bar{E}^{D}=\left[\begin{array}{ll}
1 & 0 \\
0 & 0
\end{array}\right], \quad \bar{A}^{D}=\left[\begin{array}{cc}
-2 & 0 \\
0 & -1
\end{array}\right] .
$$

In this case, the admissible initial conditions are

$$
x_{0}=\operatorname{im}\left(\bar{E} \bar{E}^{D}\right)=\operatorname{im}\left[\begin{array}{ll}
1 & 0 \\
0 & 0
\end{array}\right]=\left[\begin{array}{c}
x_{10} \\
0
\end{array}\right],
$$

and $x_{10}$ is arbitrary.

Using (9b) and 23, we obtain the nonsingular matrix

$$
\begin{aligned}
\Phi_{0}(t) & =\sum_{k=0}^{\infty} \frac{\left(\bar{E}^{D} \bar{A}\right)^{k} t^{k \alpha}}{\Gamma(k \alpha+1)} \\
& =\left[\begin{array}{ll}
1 & 0 \\
0 & 1
\end{array}\right]+\sum_{k=1}^{\infty} \frac{t^{k \alpha}}{\Gamma(k \alpha+1)}\left[\begin{array}{cc}
-0.5 & 0 \\
0 & 0
\end{array}\right]^{k} \\
& =\left[\begin{array}{cc}
1+\varphi(t) & 0 \\
0 & 1
\end{array}\right]
\end{aligned}
$$

where

$$
\varphi(t)=\sum_{k=1}^{\infty} \frac{(-0.5)^{k} t^{k \alpha}}{\Gamma(k \alpha+1)} .
$$

By Theorem 4 the positive fractional descriptor system (1) with (20) is pointwise complete for $t=t_{f}$ and every $x_{f} \in \mathbb{R}_{+}^{2}$ satisfying the condition (13). In this case, 
from (15) and (25) we have

$$
\begin{aligned}
x_{0} & =\left[\Phi_{0}\left(t_{f}\right)\right]^{-1} x_{f} \\
& =\left[\begin{array}{c}
{\left[1+\varphi\left(t_{f}\right)\right]^{-1} x_{1 f}} \\
0
\end{array}\right] \in \operatorname{im}\left(\bar{E} \bar{E}^{D}\right) \\
& =\left[\begin{array}{c}
x_{10} \\
0
\end{array}\right]
\end{aligned}
$$

if and only if

$$
x_{f} \in \operatorname{im}\left(\bar{E} \bar{E}^{D}\right)=\left[\begin{array}{c}
x_{1 f} \\
0
\end{array}\right] \in \mathbb{R}_{+}^{2},
$$

where $x_{1 f}>0$ is arbitrary.

By Theorem 5 the system (1) with (20) is pointwise degenerated in the direction $v^{T}=\left[\begin{array}{ll}0 & v_{2}\end{array}\right]$ for any $v_{2}$ since

$$
v^{T} \bar{E}=\left[\begin{array}{ll}
0 & v_{2}
\end{array}\right]\left[\begin{array}{cc}
0.5 & 0 \\
0 & 0
\end{array}\right]=\left[\begin{array}{ll}
0 & 0
\end{array}\right] .
$$

\section{Concluding remarks}

The pointwise completeness and pointwise degeneracy of positive fractional descriptor continuous-time linear systems with regular pencils have been addressed. The conditions for pointwise completeness and the pointwise degeneracy of the systems have been established (Theorems 4 and 5). The discussion has been illustrated by a numerical example of a fractional descriptor system with regular pencil. The results can be extended to positive fractional descriptor linear systems with delays.

\section{Acknowledgment}

This work was supported by the National Science Center in Poland under the grant no. N N514 638940.

\section{References}

Bru, R., Coll, C., Romero-Vivo, S. and Sanchez, E. (2003). Some problems about structural properties of positive descriptor systems, in L. Benvenuti, A. de Santis and L. Farina (Eds.), Positive Systems, Lecture Notes in Control and Information Sciences, Vol. 294, Springer, Berlin, pp. 233-240.

Bru, R., Coll, C. and Sanchez, E. (2002). Structural properties of positive linear time-invariant difference-algebraic equations, Linear Algebra and Its Applications 349(1-3): 1-10.

Busłowicz, M. (2008). Pointwise completeness and pointwise degeneracy of linear discrete-time systems of fractional order, Zeszyty Naukowe Politechniki Ślqskiej: Automatyka (151): 19-24, (in Polish).

Busłowicz, M., Kociszewski, R., Trzasko, W. (2006). Pointwise completeness and pointwise degeneracy of positive discrete-time systems with delays, Zeszyty Naukowe Politechniki Ślaskiej: Automatyka (145): 55-56, (in Polish).
Choundhury, A.K. (1972). Necessary and sufficient conditions of pointwise completeness of linear time-invariant delay-differential systems, International Journal of Control 16(6): 1083-1100.

Campbell, S.L., Meyer, C.D. and Rose, N.J. (1976). Applications of the Drazin inverse to linear systems of differential equations with singular constant coefficients, SIAM Journal Applied Mathematics 31(3): 411-425.

Dai, L. (1989). Singular Control Systems, Lectures Notes in Control and Information Sciences, Vol. 118, Springer-Verlag, Berlin.

Guang-Ren, D. (2010). Analysis and Design of Descriptor Linear Systems, Springer, New York, NY.

Farina, L. and Rinaldi, S. (2000). Positive Linear Systems: Theory and Applications, J. Wiley, New York, NY.

Kaczorek, T. and Busłowicz, M. (2009). Pointwise completeness and pointwise degeneracy of linear continuous-time fractional order systems, Journal of Automation, Mobile Robotics \& Intelligent Systems 3(1): 8-11.

Kaczorek, T. (1992). Linear Control Systems, Vol. 1, Research Studies Press J. Wiley, New York, NY.

Kaczorek, T. (2002). Positive $1 D$ and $2 D$ Systems, Springer-Verlag, London.

Kaczorek, T. (2004). Infinite eigenvalue assignment by an output feedback for singular systems, International Journal of Applied Mathematics and Computer Science 14(1): 19-23.

Kaczorek, T. (2009). Pointwise completeness and pointwise degeneracy of standard and positive fractional linear systems with state-feedbacks, Archives of Control Sciences 19(3): 295-306.

Kaczorek, T. (2010). Pointwise completeness and pointwise degeneracy of standard and positive linear systems with state-feedbacks, JAMRIS 4(1): 3-7.

Kaczorek, T. (2011a). Checking of the positivity of descriptor linear systems by the use of the shuffle algorithm, Archives of Control Sciences 21(3): 287-298.

Kaczorek, T. (2011b). Pointwise completeness and pointwise degeneracy of 2D standard and positive Fornasini-Marchesini models, COMPEL 30(2): 656-670.

Kaczorek, T. (2011c). Reduction and decomposition of singular fractional discrete-time linear systems, Acta Mechanica et Automatica 5(4): 62-66.

Kaczorek, T. (2011d). Selected Problems of Fractional Systems Theory, Springer-Verlag, Berlin.

Kaczorek, T. (2011e). Singular fractional discrete-time linear systems, Control and Cybernetics 40(3): 753-761.

Kaczorek, T. (2013). Application of Drazin inverse to analysis of descriptor fractional discrete-time linear systems with regular pencils, International Journal of Applied Mathematics and Computer Science 23(1): 29-33, DOI: 10.2478/amcs-2013-0003.

Kaczorek, T. (2014a). Drazin inverse matrix method for fractional descriptor continuous-time linear systems, Bulletin of the Polish Academy of Sciences: Technical Sciences 62(2): 409-412. 
Kaczorek, T. (2014b). Minimum energy control of positive fractional descriptor continuous-time linear systems, IET Control Theory and Applications 8(1): 219-225.

Olbrot, A. (1972). On degeneracy and related problems for linear constant time-lag systems, Ricerche di Automatica 3(3): 203-220.

Popov, V.M. (1972). Pointwise degeneracy of linear time-invariant delay-differential equations, Journal of Differential Equations 11: 541-561.

Trzasko, W., Busłowicz, M. and Kaczorek, T. (2007). Pointwise completeness of discrete-time cone-systems with delays, EUROCON, Warsaw, Poland, pp. 606-611.

Weiss, L. (1970). Controllability for various linear and nonlinear systems models, in J.A. Yorke, Seminar on Differential Equations and Dynamical Systems II, Lecture Notes in Mathematics, Vol. 144, Springer, Berlin, pp. 250-262.

Virnik (2008). Stability analysis of positive descriptor systems, Linear Algebra and Its Applications 429(10): 2640-2659.

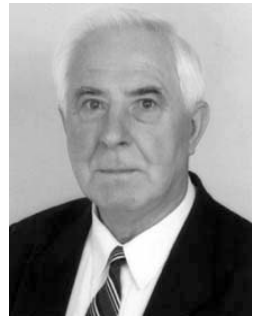

Tadeusz Kaczorek received the M.Sc., Ph.D. and D.Sc. degrees in electrical engineering from the Warsaw University of Technology in 1956, 1962 and 1964, respectively. In the years 1968-69 he was the dean of the Electrical Engineering Faculty, and in the period of 1970-73 he was a deputy rector of the Warsaw University of Technology. In 1971 he became a professor and in 1974 a full professor at the same university. Since 2003 he has been a professor at the Białystok University of Technology. In 1986 he was elected a corresponding member and in 1996 a full member of the Polish Academy of Sciences. In the years 1988-1991 he was the director of the Research Centre of the Polish Academy of Sciences in Rome. In 2004 he was elected an honorary member of the Hungarian Academy of Sciences. He was granted honorary doctorates by 13 universities. His research interests cover systems theory, especially singular multidimensional systems, positive multidimensional systems, singular positive 1D and 2D systems, as well as positive fractional $1 \mathrm{D}$ and $2 \mathrm{D}$ systems. He initiated research in the field of singular 2D, positive 2D and positive fractional linear systems. He published 28 books ( 8 in English) and over 1000 scientific papers. He also supervised $69 \mathrm{Ph} . \mathrm{D}$. theses. He is the editor-in-chief of the Bulletin of the Polish Academy of Sciences: Technical Sciences and a member of editorial boards of ten international journals.

Received: 31 January 2014 Revised: 16 April 2014 